\title{
CARCINOMA RENAL CON TRANSLOCACIÓN Xp11.2 EN UNA INFANTE
}

\author{
Xp1 1.2 TRANSLOCATION RENAL CARCINOMA IN A CHILD

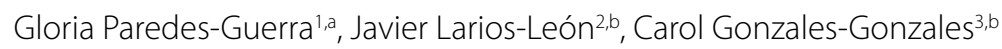

\begin{abstract}
RESUMEN
El carcinoma de células renales con translocación (CRT) es un subtipo de carcinoma de células renales (CCR). El presente caso trata de una niña de 11 años con dolor en fosa ilíaca derecha de dos semanas de evolución. Se evidenció tumoración de 2,2 × 1,9 × 2,8 cm en el polo superior del riñón derecho, por ecografía. Luego, fue sometida a nefrectomía radical derecha y a disección ganglionar regional, tras lo cual se diagnosticó CRT subtipo Xp 11.2. Posteriormente, se inició tratamiento de primera línea con pazopanib y quimioterapia concomitante.

Esta enfermedad es más frecuente entre los 10 y 15 años. Su pronóstico suele ser mejor comparado al de los adultos. El manejo multidisciplinario asociado a la disponibilidad de medicamentos de primera línea, mejora la sobrevida libre de progresión.
\end{abstract}

Palabras clave: Carcinoma de Células Renales; Carcinoma; Translocación Genética (fuente: DeCS BIREME).

\begin{abstract}
Translocation renal cell carcinoma (t-RCC) is a subtype of renal cell carcinoma (RCC).

This case is about an 11-year-old with pain in right iliac fossa for two weeks.

Tumor of $2.2 \times 1.9 \times 2.8 \mathrm{~cm}$ was evidenced in the upper pole of the right kidney, by ultrasound. Then, she underwent to a right radical nephrectomy and regional lymph node dissection, after which CRT subtype Xp 11.2 was diagnosed. Subsequently, first-line treatment with pazopanib and concomitant chemotherapy was initiated. This disease is more frequent between 10 and 15 years. Their prognosis is usually better compared to adults. The multidisciplinary management associated with the availability of first-line medications improves progression-free survival.
\end{abstract}

Key words: Carcinoma; Renal Cell; Carcinoma; Translocation; Genetic (source: MeSH NLM).

\section{INTRODUCCIÓN}

El avance en la tecnología molecular ha generado una mejor tipificación de los tipos y los subtipos de cáncer renal. En consecuencia, hoy en día se reconocen los factores pronósticos y predictivos, por lo que se llega a aumentar la sobrevida específica por cáncer.

Los carcinomas de células renales son neoplasias muy raras en niños. Por lo general, son adultos mayores de 50 años quienes los padecen. Su incidencia en niños se estima entre $1,8 \%$ y $6,3 \%$, y su frecuencia es directamente proporcional a la edad ${ }^{(1)}$.

En el año 2016, la clasificación de tumores genitourinarios de la Organización Mundial de la Salud (OMS) agregó, dentro de los subtipos de carcinomas de células renales, a los carcinomas de células renales por translocación de la familia MiT, cuyo estudio molecular abarca la translocación Xp11.2 y la translocación $\mathrm{t}(6 ; 11)$, cada una con características clínicas y pronósticos variables ${ }^{(1,2)}$.

\footnotetext{
${ }^{1}$ Clínica Internacional, Lima-Perú.

${ }^{2}$ Servicio de Oncología Médica, Hospital Nacional Almanzor Aguinaga Asenjo, Chiclayo-Perú.

${ }^{3}$ Hospital de Alta Complejidad Virgen de La Puerta, Trujillo-Perú.

a Oncólogo pediatra.

b Residente de oncología médica.

Citar como: Gloria Paredes-Guerra, Javier Larios-León, Carol Gonzales-Gonzales. Carcinoma renal con translocación Xp11.2 en una infante. Rev. Fac. Med. Hum. Enero 2020; 20(1):153-157. DOI 10.25176/RFMH.v20i1.2556 
El manejo de los carcinomas de células renales con translocación no suele variar con respecto a otros subtipos de carcinomas de células renales. La cirugía es el tratamiento principal para este tipo de neoplasias, independientemente del hallazgo de metástasis al inicio de la enfermedad. Por otro lado, el uso de medicamentos diana, tales como sunitinib o pazopanib, ambos inhibidores de tirosina quinasa, han contribuido a una mejora de la sobrevida global y la sobrevida libre de progresión de enfermedad en estos pacientes. El manejo interdisciplinario y la disponibilidad de estos medicamentos son fundamentales para asegurar una larga sobrevida en niños portadores de carcinoma de células renales con translocación ${ }^{(1,2)}$.

El caso que se expone en el presente informe resalta la larga sobrevida libre de progresión de una niña con carcinoma de células renales con translocación Xp 11.2, determinado por hibridación fluorescente in situ (FISH, por su sigla en inglés).

\section{PRESENTACIÓN DEL CASO}

Se detalla el caso de una niña de 10 años de edad, que refería astenia, palidez y dolor de tipo "punzada" a nivel de fosa iliaca derecha, no irradiado, de dos semanas de evolución, por lo cual acude a emergencia de la clínica en noviembre del 2016.

Sus antecedentes personales son los siguientes: hematoma renal postraumático de $2,2 \times 1,9 \times 2.8 \mathrm{~cm}$ en abril del 2013.

Al examen físico, se observa a una paciente con palidez moderada $++/+++$, sin signos de sangrado activo, con dolor a la palpación superficial y a la palpación profunda a nivel de la fosa ilíaca y flanco derecho, sin evidencia de tumoraciones palpables a nivel abdominal. Resto del examen no contributorio.

Se inicia un tratamiento analgésico con leve mejoría del dolor y se realiza una ecografía renal, hallando, en el polo superior del riñón derecho, una imagen sólida ecogénica con centro hipoecogénico de bordes regulares de 33,6 × 22,7 mm, sin vascularidad interna.

Asimismo, es evaluada por el urólogo, quien solicita que se adjunte una resonancia magnética de abdomen al plan de trabajo, donde se visualiza una imagen nodular de 2,2 x 1,9 x 2,8 cm $\neg$-que compromete el polo superior del riñón derecho--, contornos lobulados sin signos infiltrativos, hiposeñal en T2 y zona central hiperintensa, con leve captación al contraste, compatible con tumor renal.

En el estudio de extensión con tomografía axial computarizada de tórax, se evidencia un conglomerado ganglionar en el recorrido de la vena ácigos y en el grupo paraesofágico derecho. Además, en la analítica, se encontraron $480 \mathrm{ng} / \mathrm{dl}$ de deshidrogenasa láctica, 9,3 $\mathrm{gr} / \mathrm{dl}$ de hemoglobina, $9 \mathrm{mg} / \mathrm{dl}$ de calcio sérico, 4200 neutrófilos y 220000 plaquetas.

Cuatro días después, la paciente fue estabilizada y sometida a una biopsia renal percutánea, y el resultado anatomopatológico fue neoplasia renal de células eosinófilas.

Ante este diagnóstico se decide someter a la paciente a nefrectomía radical derecha asociada a linfadenectomía regional. En los hallazgos anatomopatológicos se describe un tumor de color vinoso de aproximadamente $8 \times 6,5 \times 6 \mathrm{~cm}$, de consistencia dura. Al microscopio, se encuentra una neoplasia maligna renal de patrón predominantemente papilar, constituida por células de aspecto epitelioide, con alto grado nuclear, citoplasma abundante eosinofílico, que compromete seno renal, necrosis tumoral presente pero mínima, márgenes quirúrgicos libres de neoplasia, invasión linfovascular presente, 22 ganglios linfáticos resecados, de los cuales 7 estaban infiltrados por neoplasia maligna.

Para complementar, se realizan las siguientes pruebas inmunohistoquímicas: AMACR (+), CD10 (+), EMA (-), Ki 67\%: 15\%, CK 7 (-), cuyo resultado es carcinoma de células renales por translocación.

Se realiza el estudio genético al tumor, según la prueba FISH, y se confirma el diagnóstico de carcinoma de células renales con translocación (CRT), con un resultado positivo para la translocación Xp 11.23/17q25.3 (tabla y figura 1).

Tabla 1. Resultado final de la prueba FISH.

\section{Resultado}

Translocación Xp11.23/17q25.3: Positivo para la translocación en 83\% de las células analizadas

Translocación Xp11.23/1q23.1: Negativo para la translocación en 100\% de las células analizadas 


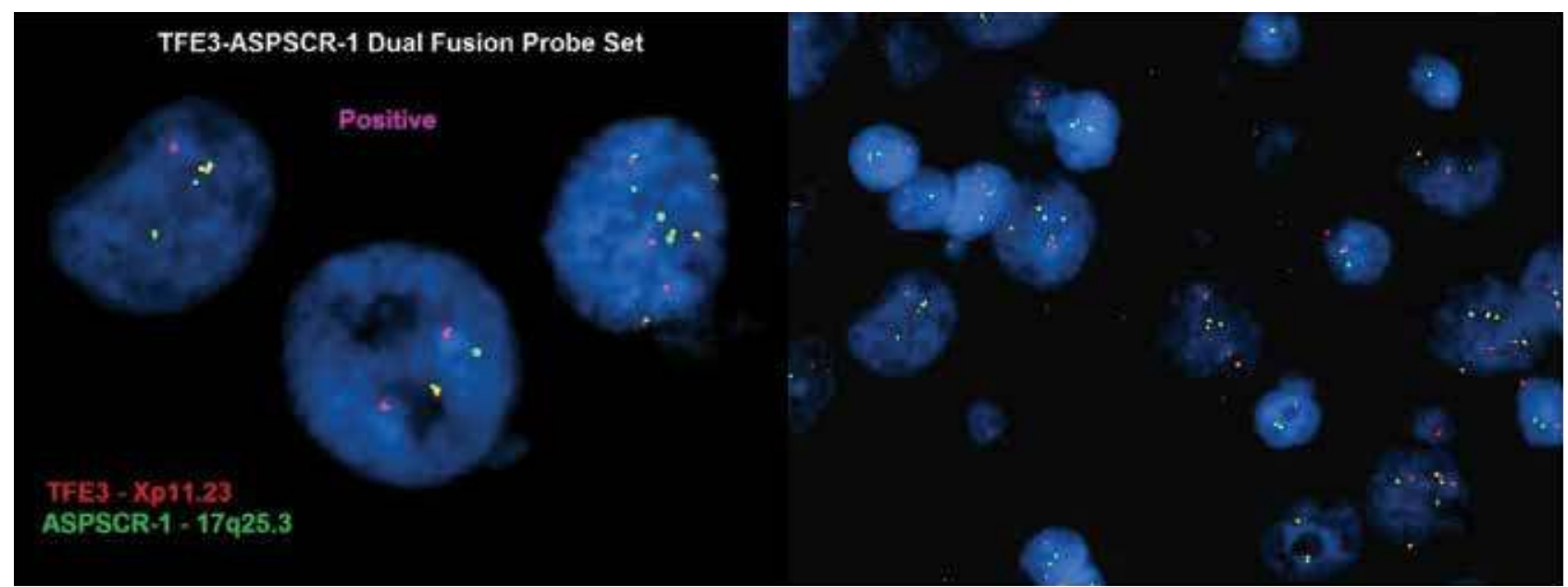

Figura 1. Imágenes de la prueba FISH que dio positivo para la translocación Xp11.2.

Al confirmarse el diagnóstico de carcinoma de células renales por translocación Xp 11.2, la paciente fue tipificada según el Internacional Metastatic Renal Cell Carcinoma Database Consortium (IMDC, por su sigla en inglés) que se muestra en la tabla 2. Luego, se obtuvo un score de dos puntos (considerando un tiempo desde el diagnóstico hasta el inicio del tratamiento menor de un año y una hemoglobina por debajo del límite inferior normal). Finalmente, se estimó que la paciente pertenecía al grupo de riesgo intermedio.

Tabla 2. Clasificación IMDC usada para tipificar a la paciente luego de la nefrectomía radical.

\section{Factores pronósticos}

\section{Grupos de riesgo}

1) Tiempo transcurrido desde el diagnóstico hasta

el inicio del tratamiento menor de un año

2) Karnofsky $<80 \%$

3) Emoglobina por debajo del límite inferior normal (normal: $12 \mathrm{~g} / \mathrm{dl}$ )

4) Calcio por encima del límite superior normal (normal: 8,5-10,2 mg/dl)

5) Neutrófilos por encima del límite superior normal

1. Grupo de bajo riesgo: ningún factor pronóstico.

2. Grupo de riesgo intermedio: $0-2$ factores pronósticos

3. Grupo de alto riesgo: $>3$ factores pronósticos (normal: 2,0-7,0 x 103/l)

6) Plaquetas por encima del límite superior normal (normal: 150 000-400 000)

La paciente inicia pazopanib a $400 \mathrm{mg} /$ día durante tres meses. Se realiza un control tomográfico de torax, abdomen y pelvis, y se observa que las lesiones metastásicas mediastinales persisiten, asociando quimioterapia con esquema cisplatino $75 \mathrm{mg} / \mathrm{m} 2$ en $\mathrm{D} 1$ + gemcitabine $1000 \mathrm{mg} / \mathrm{m} 2$ en D1 y D8, cada veintiocho días por un total de seis ciclos. Se logra una respuesta parcial a nivel de conglomerado ganglionar mediastinal.

Ocho meses después del diagnóstico la paciente es sometida a metastasectomía ganglionar mediastinal parcial (debido a presentación paraesofágica), cuyo estudio anatomopatológico confirma metástasis de carcinoma de células renales por translocación Xp 11.2.

Debido a la persistencia de la masa residual ganglionar paraesofágica, se prescribe el inicio de sunitinib, medicamento que los padres rehusan a administrarle, por lo que la paciente continúa con controles periódicos clínicos e imagenológicos donde se evidencia la persistencia estable de esa lesión y una escala de Zubrod de cero. 
Hasta el momento de la publicación, la paciente tiene una sobrevida libre de enfermedad de aproximadamente dieciocho meses, con controles periódicos de tomografías para evaluar la progresión de enfermedad.

\section{DISCUSIÓN}

Los carcinomas de células renales son neoplasias muy raras durante la infancia, representando tan solo del $2 \%$ al $3 \%$ de los tumores renales malignos en niños. Sin embargo, se ha demostrado que su incidencia incrementa con la edad ${ }^{(1)}$.

De acuerdo con la Japanese Society of Pediatric Surgeons, los carcinomas de células renales representan el 1,4\% de todos los tumores renales en niños menores de 4 años, el 15,2\% en pacientes entre los 5 y 9 años, y el $52,6 \%$ en pacientes entre los 10 y 15 años, teniendo como pico de incidencia los 9 y 15 años ${ }^{(2)}$.

En el año 2016, la OMS incluyó, dentro de los tumores de células renales, el subtipo carcinoma de células renales con translocación de la familia MiT, el cual comprende el carcinoma de células renales con translocación Xp11 y el carcinoma de células renales con translocación $\mathrm{t}(6 ; 11)$.

Es fundamental establecer que los subtipos del CRT $\mathrm{Xp} 11$ y $\mathrm{t}(6 ; 11)$ son causados por reordenamientos en los genes de los factores de transcripción TFE3 y TFEB, respectivamente ${ }^{(3,4)}$.

Determinar la frecuencia exacta de los CRT es un desafío. Algunos autores como Kmetec et al. ${ }^{(5)}$ han determinado que los CRT Xp11 representan el 20-40\% de los CCR en niños, mientras que la data ofrecida por Komai et al. ${ }^{(6)}$. establece que, aproximadamente, un tercio de los CCR en niños será CRT. Esta diferencia se debe principalmente a la rareza del tumor, de tal manera que las características clínico-patológicas, pronóstico y tratamiento han sido obtenidas de datos retrospectivos.

El diagnóstico de los carcinomas de células renales suele determinarse por morfología, mientras que el de los carcinomas de células renales con translocación exige la comprobación de los reordenamientos en los factores de transcripción TFE3 y TFEB, para lo cual se emplea, generalmente, la técnica citogenética de marcaje de cromosomas, mediante la cual estos se hibridan con sondas que emiten fluorescencia $(\mathrm{FISH})^{(7,8)}$.

Se presentó el caso de una niña de 11 años, con un diagnóstico de carcinoma de células renales con translocación Xp11, en estadio IV por compromiso ganglionar a distancia. Fue sometida a nefrectomía radical derecha y a linfadenectomía regional, y, posteriormente, inició un tratamiento de pazopanib de 400 mg/día asociado a quimioterapia basada en platino (cisplatino de $75 \mathrm{mg} / \mathrm{m} 2$ en D1 + Gemcitabine 1000 mg/m2 en D1 y D8) cada 28 días por un total de seis ciclos, lo cual permitió que la paciente sea sometida a resección ganglionar mediastinal y que mantenga una sobrevida libre de progresión de aproximadamente 18 meses.

Sukov et al. publicaron los resultados de 632 pacientes con CCR, de los cuales solo seis demostraron ser portadores de reordenamientos de TFE3. Los investigadores establecieron que los pacientes debutan con un compromiso ganglionar asociado a una enfermedad de comportamiento agresivo. Asimismo, el estudio resaltó una peor sobrevida libre de progresión (SLP) para aquellos pacientes portadores de reordenamientos de TFE3 en comparación con aquellos que no eran portadores ${ }^{(9,10)}$.

La sobrevida global reportada para los niños con CCR varía de acuerdo con los siguientes factores: 1) la extensión de la cirugía (nefrectomía radical +/disección ganglionar regional), 2) estadio clínico al debut, 3) disponibilidad de medicamentos de primera línea en enfermedad metastásica y 4) seguimiento estricto del paciente. Indolfi $P$. et al. realizaron un análisis retrospectivo de 41 pacientes, entre niños y adolescentes, con el cual demostraron que los pacientes con CCR tienen, en promedio, una tasa de sobrevida global de $63 \%$, con tasas de sobrevida determinadas por el estadio clínico. Además, señalaron que entre los estadios I y IV hay una tasa de sobrevida de $92,4 \%, 84,6 \%, 72,7 \%$ y $13,9 \%$, respectivamente ${ }^{(11)}$.

Es difícil determinar con exactitud la sobrevida global y la sobrevida libre de progresión en niños con carcinoma de células renales con translocación Xp11.2, debido a que no existe un estudio prospectivo en este grupo de población, lo que dificulta su comparación con otros subtipos de carcinoma de células renales. Asimismo, se ha establecido que el pronóstico de los carcinomas de células renales con translocación Xp11.2 en niños es mejor comparado al de adultos ${ }^{(5,12)}$.

Al-Daghmin et al. realizaron una revisión retrospectiva de 23 pacientes con carcinoma de células renales con translocación Xp 11.2, incluyendo solamente dos pacientes menores de 18 años (dos niñas de trece y dos años, respectivamente), y llegaron a la conclusión de que, luego de un seguimiento de 35 meses, la tasa de sobrevida libre de enfermedad en 3 años fue del $75 \%{ }^{(13)}$.

La paciente presentada fue tratada según lo establecido por las guías internacionales, y obtuvo una 
sobrevida libre de progresión de, aproximadamente, 18 meses hasta la actualidad. Todo ello nos permite establecer que un adecuado manejo interdisciplinario inicial logrará una larga sobrevida libre de enfermedad en estos pacientes.

\section{CONCLUSIÓN}

El manejo multidisciplinario desde un inicio asociado a la disponibilidad de medicamentos de primera línea para este tipo de neoplasias mejora significativamente la sobrevida libre de progresión en niños portadores de carcinoma de células renales por translocación.
Contribuciones de autoría: Los autores participaron en la génesis de la idea, diseño de proyecto, recolección e interpretación de datos, análisis de resultados, preparación del manuscrito.

Financiamiento: Autofinanciado.

Conflicto de interés: Los autores declaran no tener ningún conflicto de interés.

Recibido: 10 de octubre del 2019

Aprobado: 13 de diciembre del 2019

Correspondencia: Gloria Paredes Guerra.

Dirección: Av. Inca Garcilaso de la Vega 1420, Cercado de Lima, Lima-Perú. Teléfono: 981291940

Correo: gloriaparedes2000@yahoo.com

\section{REFERENCIAS BIBLIOGRÁFICAS}

1.- Indolfi P, Terenziani M, Casale F, Carli M, Bisogno G, Schiavetti A, et al.: Renal cell carcinoma in children: A clinicopathologicstudy. J Clin Oncol. 2003;21(3):530-5. https://doi.org/10.1200/JCO.2003.02.072

2. - Xian P, Jing Q, Liwen Z, Wenhua L. Xp11.2 translocation renal cell carcinoma with TFE3 gene fusión: A case report. Molecular and clinical oncology 8: 83-85, 2018. https://doi.org/10.3892/mco.2017.1497

3. - Moch H, Cubilla AL, Humphrey PA, Reuter VE, Ulbright TM. The 2016 WHO Classification of Tumours of the Urinary System and Male Genital Organs-Part A: Renal, Penile, and Testicular Tumours. Eur Urol 2016;70:93-105. https://doi. org/10.1016/j.eururo.2016.02.029.

4. - Ellati RT, Abukhiran I, Alqasem K, Jasser J, Khzouz J, Bisharat T, Al Saidi I and Al Daghmin A: Clinicopathologic features of translocation renal cell carcinoma. Clin Genitourin Cancer 15: 112 116, 2017. https://doi.org/10.1016/j. clgc.2016.05.013

5. Kmetec A and Jeruc J: Xp 11.2 translocation renal carcinoma in young adults; recently classified distinct subtype. Radiol Oncol 48: 197 202, 2014. https://dx.doi.org/10.2478\%2Fraon-2013-0077

6. Komai $Y$, Fujiwara $M$, Fujii $Y$, Mukai $H$, Yonese J, Kawakami $S$, et al. Adult Xp11 translocation renal cell carcinoma diagnosed by cytogenetics and immunohistochemistry. Clin Cancer Res 2009;15:1170-6. https://doi. org/10.1158/1078-0432.CCR-08-1183.

7. Inamura K. Translocation Renal Cell Carcinoma: An Update on Clinicopathological and Molecular Features. Cancers (Basel) 2017;9:E111. https://doi.org/10.3390/cancers9090111.
8. Ellis CL, Eble JN, Subhawong AP, Martignoni G, Zhong M, Ladanyi M, et al. Clinical heterogeneity of Xp11 translocation renal cell carcinoma: impact of fusion subtype, age, and stage. Mod Pathol 2014;27:875-86. https://doi. org/10.1038/modpathol.2013.208.

9. Sukov WR, Hodge JC, Lohse CM, Leibovich BC, Thompson RH, Pearce $\mathrm{KE}$, et al. TFE3 rearrangements in adult renal cell carcinoma: clinical and pathologic features with outcome in a large series of consecutively treated patients. Am J Surg Pathol 2012;36:663-70. https://doi.org/10.1097/ PAS.0b013e31824dd972..

10. Abdellah A, Selma K, Elamin M. Renal cell carcinoma in children: case report and literatura review. PanAfrican Medical Journal. 2015: 1-4. https://doi. org/10.11604/pamj.2015.20.84.5791

11. Rialon $K$, Gulack B, Englum B. Factors impacting survival in children with renal cell carcinoma. J Pediatr Surg. 2015; 50(6): 1014-1018. https://doi. org/10.1016/j.jpedsurg.2015.03.027

12. Klatte T, Streubel B, Wrba F, Remzi M, Krammer B, de Martino M, et al. Renal cell carcinoma associated with transcription factor E3 expression and Xp11.2 translocation: incidence, characteristics, and prognosis. Am J Clin Pathol 2012;137:761-8. https://doi.org/10.1309/AJCPQ6LLFMC4OXGC..

13. Al-Daghmin A, Alhamss S, Al-qasem K, Al-najjar H, Al-smadi K, Olaimat A, et al. Patterns of management of translocation renal cell carcinoma. Turk J Urol 2018; 44(6): 467-72. https://doi.org/10.5152/tud.2018.40460

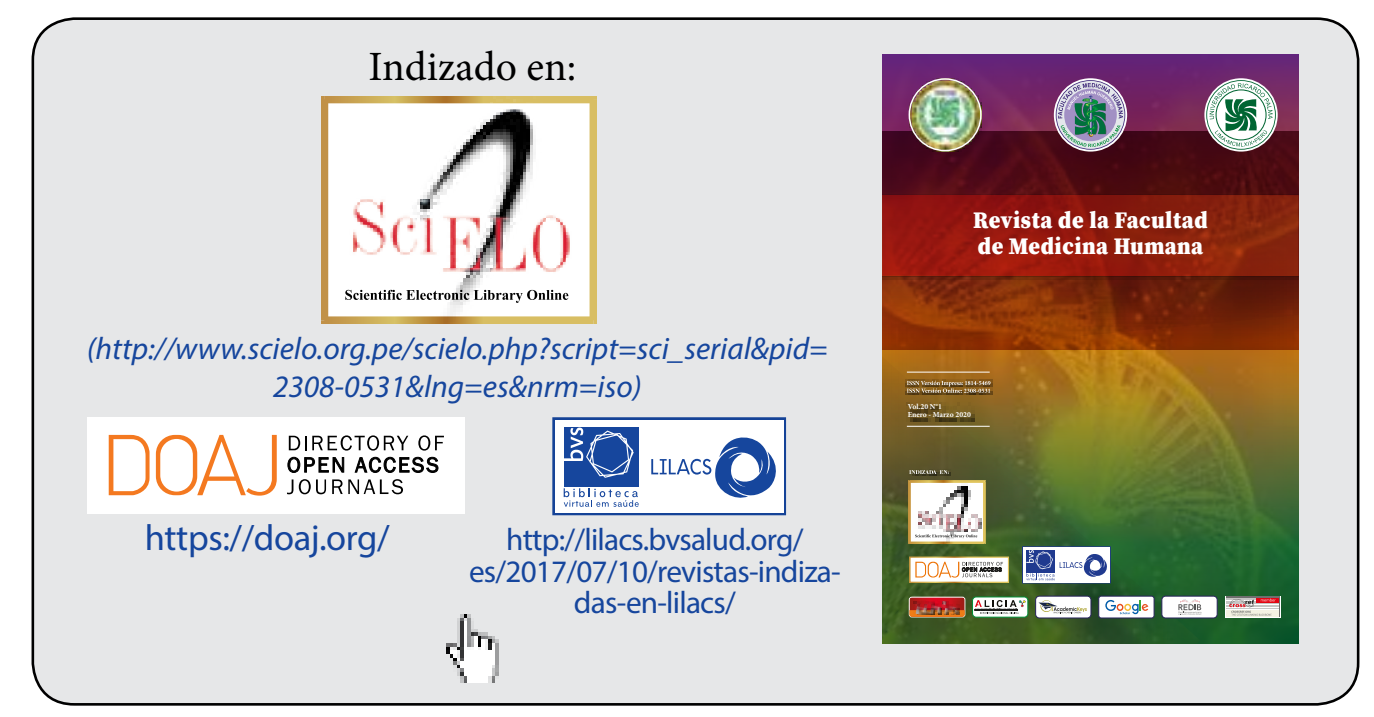

